\title{
Truncated Hybrid Double Acceptance Sampling Plan (THDASP) for Weibull Product Life Distribution
}

\author{
Braimah Odunayo Joseph ${ }^{1}$, Osanaiye Peter Asanaiye ${ }^{2}$ \\ ${ }^{1}$ Department of Mathematics, Ambrose Alli University, Ekpoma, Nigeria \\ ${ }^{2}$ Department of Statistics, University of Ilorin, Ilorin, Nigeria
}

Email address:

ojbraimah2014@gmail.com (B. O. Joseph)

\section{To cite this article:}

Braimah Odunayo Joseph, Osanaiye Peter Asanaiye. Truncated Hybrid Double Acceptance Sampling Plan (THDASP) for Weibull Product Life Distribution. American Journal of Management Science and Engineering. Vol. 2, No. 5, 2017, pp. 80-88. doi: 10.11648/j.ajmse.20170205.12

Received: March 20, 2017; Accepted: April 17, 2017; Published: October 23, 2017

\begin{abstract}
In this paper, an improved reliable acceptance sampling plan (Truncated hybrid Double Acceptance Sampling Plan (THDASP)) is proposed for products life that follows Weibull distribution when the testing is truncated at a specified time (t). This type of inspection sampling plan can be used to save the testing time in practical situations. The optimal sample sizes (n) required for testing product quality to ascertain a true mean life is obtained under a given Maximum Allowable Percent Defective $(\beta)$, test termination ratios and acceptance numbers $(\mathrm{C})$. The operating characteristic (OC) values formula is being developed considering both the Producer's and Consumer's risk and the values are generated. The Mean Life Ratios and curves of the plan are examined with varying ratio of the true mean life to the specified life. The advantage of this inspection plan is that could it results in better economic reliability product quality testing that protects the producer from rejecting his good lots and consumers from accepting bad lots of finished products. The mean life ratio values will also guides the producer on how to improve on his product's quality. A numerical example is also discussed for illustrative purpose.
\end{abstract}

Keywords: Truncated, Acceptance Sampling, Reliability, Producer's Risk, Consumer's Risk, Mean Ratio, Operating Characteristics

\section{Introduction}

The purpose of acceptance sampling is illustrated in this paper. For instance, if a company receives a delivery of product from a merchant, this product is always a component or raw material used in the company's manufacturing process. A sample is taken from the lot and the relevant quality characteristic of the units in the sample is inspected, Srinivasa (2011). Base on the information in this sample, a decision is made regarding lot outlook. Usually, when the life test indicates that the mean life $(\mu)$ of products exceeds the specified $\left(\mu_{\mathrm{o}}\right)$ one, the lot of products is accepted, otherwise it is rejected. Accepted lots are put into production, while rejected lots may be returned to the merchant or may be subjected to some other lot disposition action. While it is usual to think of acceptance sampling as a receiving inspection activity, there are also other uses. Frequently, a manufacturer samples and inspects its own product at various stages of production. Lots that are accepted are sent forward for further processing, while rejected lots may be reworked or scrapped. For the purpose of reducing the test time and cost, a truncated life test may be conducted to determine the smallest sample size to ensure a certain mean life of products when the life test is terminated at a pre-assigned time $t$ and the number of failures observed does not exceed a given acceptance number $c$.

Acceptance sampling is concerned with inspection and decision making regarding lots of product and constitutes one of the oldest techniques in quality assurance. Sampling plans is used to determine the acceptability of lots of items, Priyah and Ramaswamy (2015). Life test refers to measurements of product life; product life can be measured in hours, miles, cycles or any other metric that applies to the period of successful operation of a particular product. Since time is a common measure of life, life data 
points are often called times-to-failure. There are different types of life products. Statistical distributions have been assumed by various authors (statisticians, mathematicians and engineers) to mathematically model or represent certain behaviour of products. The probability density function (pdf) and cumulative distribution function (cdf) are mathematical functions that explain the distribution of life of an item.

Epstein (1954) and Sobel and Tischendrof (1959) were first to discussed acceptance sampling based on truncated life tests for an exponential model. An extensionof their work was carried out in Goodeand Kao (1961). by considering the Weibullmodel which includes the exponential distribution. Gupta $(1960,1962)$ also considered the gamma and log-normal distributions respectively. Recently Balakrishnan et al. (2007) discussed acceptance sampling based on the inverse Rayleigh distribution, Muhammad et al (2010) discussed time truncated acceptance sampling plans for generalized exponential distribution, Sudamani, and Jayasri (2012) discussed time truncated chain sampling plans for generalized exponential distribution, Sudamani and Pyrah (2012) discussed acceptance sampling plan for truncated life tests at maximum allowable percent defective. Lastly, Sudamani and Jayasri (2013) discussed time truncated chain sampling plans for Marshall-Olkin extended exponential distributions.

In this paper, we developed a truncated hybrid double acceptance sampling plan by considering both the producers and consumers' risk, which has single sampling plan as attribute plan to obtain the test termination ratios, assuming that the life time of the product follows a Weibull distribution.

\subsection{Proposed Truncated Hybrid Double Sampling Plan (THDSP)}

Sherman (1965) proposed the attributes repetitive sampling plan for a normal distribution. The procedure of this repetitive sampling is like that of sequential sampling. According to his study, his technique gives the minimum sample size with the required protection to consumer. In addition, repetitive sampling is efficient than single sampling plan. Various authors as well as Balamurali and Jun (2006) discussed on variables repetitive acceptance sampling scheme and compared the results with the single sampling scheme. Nevertheless, no attempt has been made to study attributes hybrid sampling plans based on truncated life tests. In this paper, we attempt to propose and developed an attributes truncated hybrid double sampling plan with known quality parameter and that the product quality level is represented by the ratio of mean life to the specified life $\left(\frac{\mu}{\mu_{o}}\right)$.

The double sampling plan entails two sample sizes $\left(\mathrm{n}_{1}\right.$ and $\mathrm{n}_{2)}$ and also requires two acceptance numbers $\left(\mathrm{c}_{1}\right.$ and $\left.\mathrm{c}_{2}\right)$. In all reviewed literature of double sampling plans including Aslam et al. (2009), they only considered the case of $c_{1}=0$ and $c_{2}=1$.The design parameters they considered was only the consumer's risk. But, in our present study, we consider both the producer's and consumer's risk at the same time when determining the design parameters which is quite different from the work of Aslam et al. (2009).

In order to determine the design parameters of our proposed truncated hybrid double sampling plan (THDASP), we choose the approach based on two points on the Operating Characteristics (OC) curve by considering both the producer's and consumer's risks. Several authors, like Aslam and Jun (2009), developed their own sampling plans using this point of view.

Our approach measures the quality level of a product through the ratio of its true mean lifetime to the specified length $\left(\mu / \mu_{o}\right)$. These mean life ratios enables the producer to improve the quality of his products. From the producer's point of view, the lot acceptance probability should not be less than 1- $\alpha$ at the Acceptable Quality Level (AQL). Therefore, the producer stressed that a lot should be accepted at various levels, $\left(\mu / \mu_{0}=2,4,6,8,10\right.$ and 12). Conversely, from the consumer's point of view, the probability of lot rejection should be at most $\beta$.

\subsubsection{Operating Procedure for the Proposed Truncated Hybrid Double Sampling Plan}

Our proposed attributes truncated hybrid double acceptance sampling plan can be illustrated as follows:

i. Select a random sample of sizenfrom a lot andput them to life test at a prefixed time $t$.

ii. Accept the lot if the number of defective or failed items (d) isless than or equal to the first acceptance number $\left(c_{1}\right)$. Truncate or stop the test and reject the product lot as soon as the number of defective items (d) is more than the second acceptance number $\left(\mathrm{c}_{2}\right)$.

iii. Whenever $c_{1}<d \leq c_{2}$, then Step 1 is repeated.

Note: Under our proposed plan, $n_{1}=n_{2}=\operatorname{nand}_{1} \leq c_{2}$.

\subsubsection{Advantages of the Proposed Truncated Hybrid Double Sampling Plan over Ordinary Truncated Double Sampling}

i. It is easier to design, administer and explain compare to ordinary double sampling plan.

ii. The sample size from lots to lots is constant (i.e, it is characterized with, $\mathrm{C}_{1}, \mathrm{C}_{2}$, and $\mathrm{n}$ ).

iii. Since the sample size is constant, the time and cost of inspection because taking a higher sample might incur more testing cost and time; thereby psychologically making the Producer not satisfied.

iv. The design parameters were obtained by putting bother the producer's and consumer's risk into consideration.

The flow chart of the proposed repetitive truncated double sampling scheme is as shown in figure 1 below: 


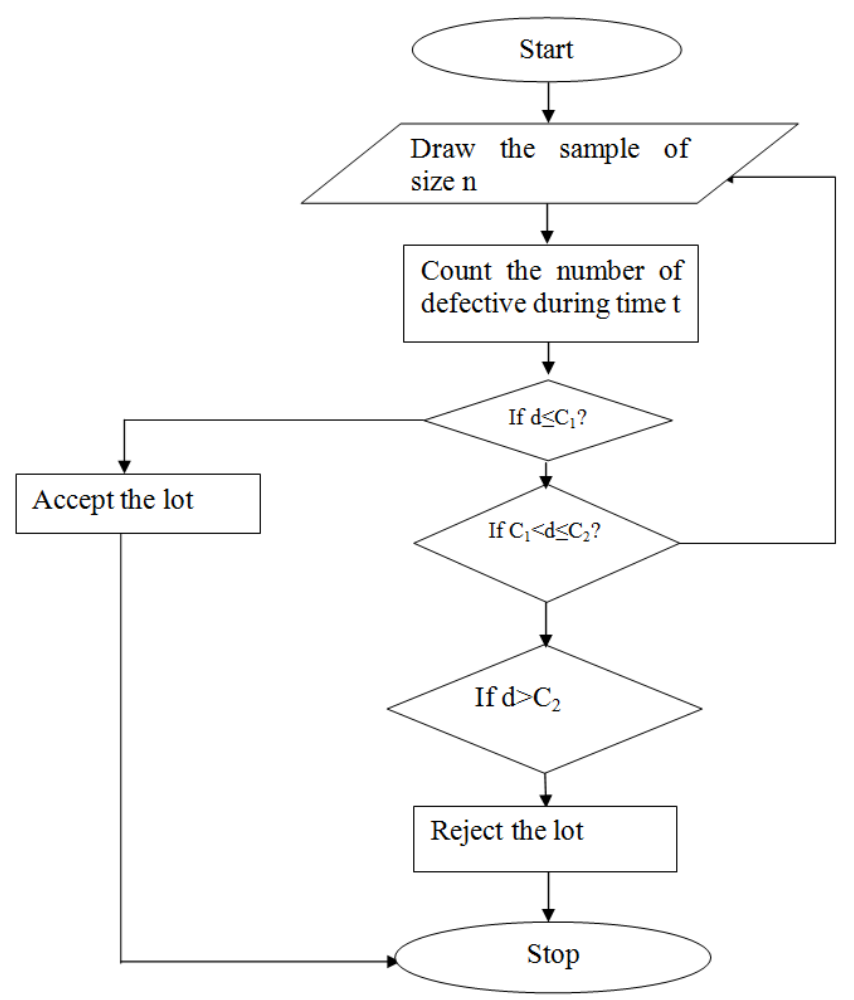

Figure 1. Flow chart process for the proposed truncated hybrid double acceptance sampling plan.

\subsection{Weibull Distribution}

The Weibull distribution is the generalization of the exponential distribution. The distribution was proposed by Weibull in 1939 and is widely applied in failure situations. The Weibull is one of the most popular distributions for analyzing lifetime data. This distribution has been studied in the several literatures and has applications in fields other than lifetime distributions, Goode and Kao (1961). The Weibull analysis has an advantage in that it has the ability to provide reasonably accurate failure analysis and failure forecast with extremely small samples. Another advantage of this distribution is that it provides a simple and useful graphical plot of failure data, Priyah and Ramaswamy (2015). Solutions are possible at the earliest indications of a problem. Small samples also allow cost effective component testing. For example, sudden death Weibull tests are completed when the first failure occurs in each lot of components (for example, lots of ball bearings). If all the bearings are tested to failure, the cost and time required is much greater.

Another advantage of Weibull distribution is that it provides a simple and useful graphical plot of the failure data. The data plot is extremely important to the engineer and to the manager. Marshall and Olkin (1997) stated that the Weibull distribution is applicable to many survival and reliability analysis with decreasing, increasing and hazard rate. The three parameters distribution, represent location, scale and shape, and because of them, it is quite a bit of flexible for analyzing skewed data. The Weibull distribution has become one of the most commonly used lifetime distributions in reliability engineering and elsewhere due to its versatility and relative simplicity. It is a flexible distribution that can take on the characteristics of other types of distributions, based on the value of the shape parameter. The Weibull distribution is very popular among engineers. One reason for this is that the Weibull cumulative distribution function (cdf) has a closed form. The Weibull distribution function is defined as:

$$
F(\alpha, \mu, x)=1-\exp \left[-\left(\frac{x}{\alpha}\right)^{\mu}\right]
$$

The parameters $\mu>0$ and $\alpha>0$ are referred to as scale and shape parameter, respectively. The Weibull density has the following form:

$$
\begin{gathered}
f(\alpha, \mu, x)=F^{\prime}(\alpha, \mu, x)=\frac{d}{d x} F(\alpha, \mu, x)= \\
\frac{\mu}{\alpha}\left(\frac{x}{\alpha}\right)^{\mu-1} \exp \left[-\left(\frac{x}{\alpha}\right)^{\mu}\right]
\end{gathered}
$$

If $\mu=1$, the Weibull distribution coincides with the exponential distribution with mean $\alpha$ densities.

The mean and variance of a Weibull distributionis given as:

$$
E(X)=\alpha \Gamma\left(1+\frac{1}{\mu}\right)
$$

and

$$
\sigma^{2}=\alpha^{2}\left[\Gamma\left(1+\frac{2}{\mu}\right)-\left\{\Gamma\left(1+\frac{1}{\mu}\right)\right\}^{2}\right]
$$

When the shape parameter $\mu$ in a Weibull distribution is given any fixed value, it reduces to one parameter weibull distribution. If $\mu=2$, it reduces to Rayleigh distribution and if the scale parameter $\alpha=1$, the Weibull distribution reduces to exponential distribution. In time truncated acceptance sampling plan, the cumulative distribution function as recently used in Braimah and Osanaiye (2016) is given as:

$$
F(t, \mu)=1-e^{-\left(\frac{t}{\mu_{0}}\right)^{\alpha}}
$$

where $\mu$ is the scale parameter (quality parameter) and $\alpha$ is the shape parameter.

\section{Methods}

\subsection{Development of Operating Characteristics for Proposed Hybrid Double Acceptance Sampling Plan}

An acceptance sampling plan is best described in graphical terms on an operating characteristic curve (OC curve). An OC curve is a plot of the actual number of nonconforming units in a lot (expressed as a percentage) against the probability that the lot will be accepted when sampled according to the plan. The shape of an OC curve is determined primarily by sample size, n, and acceptance number, c, although there is a small effect of lot size $(\mathrm{N})$. The OC function of the sampling plan $\left(n, c, \frac{t}{\mu_{0}}\right)$ is the probability ofaccepting a lot and is given by

$$
L(P)=\sum_{i=0}^{c}\left(\begin{array}{c}
n \\
i
\end{array}\right) p^{i}(1-p)^{n-1}
$$


The above proposed truncated hybrid double acceptance sampling plan is described by three parameters $\left(n, \mathrm{c}_{1}\right.$ and $\left.\mathrm{c}_{2}\right)$. It is of a note that this Truncated Hybrid Double Sampling Plan (THDSP) is a generalization of the truncated single acceptance sampling plan and becomes single plan when $\mathrm{c}_{1}=$ $\mathrm{c}_{2}$. The probability of lot acceptance $(\mathrm{Pa})$ is obtained by adopting and modification using the Operating Characteristic (OC) function of double sampling plan, which is derived as follows:

$$
\text { Suppose } P_{a}\left(p_{1}\right)=\sum_{\mathrm{i}=0}^{\mathrm{c}_{1}}\left(\begin{array}{c}
\mathrm{n} \\
\mathrm{i}
\end{array}\right)(1-p)^{\mathrm{n}-1}
$$

is the probability of acceptance of a submitted lot with fraction defective $p$ based on a given sample with the first acceptance number and

$$
P_{a}\left(p_{2}\right)=\sum_{i=0}^{c_{2}}\left(\begin{array}{c}
\mathrm{n} \\
i
\end{array}\right) p^{i}(1-p)^{\mathrm{n}-\mathrm{i}}
$$

be the corresponding probability of acceptance of a submitted lot with fraction defective $p$ based on a given sample with the second acceptance number. We then define the corresponding probability of lot rejection as:

$$
\operatorname{Pr}(\mathrm{p})=1-\sum_{\mathrm{i}=0}^{\mathrm{c}_{2}}\left(\begin{array}{l}
\mathrm{n} \\
\mathrm{i}
\end{array}\right) \mathrm{p}^{\mathrm{i}}(1-\mathrm{p})^{\mathrm{n}-\mathrm{i}}
$$

These probabilities are therefore given by:

$$
P(a)=\operatorname{Pr}\left(\mathrm{d} \leq \mathrm{c}_{1}: \mathrm{p}\right)=\sum_{\mathrm{i}=0}^{\mathrm{c}_{1}}\left(\begin{array}{l}
\mathrm{n} \\
\mathrm{i}
\end{array}\right)(1-p)^{\mathrm{n}-\mathrm{i}}
$$

and the corresponding probability of lot rejection

$$
P(r)=\operatorname{Pr}\left(d>c_{2}: p\right)=1-\sum_{i=0}^{c_{2}}\left(\begin{array}{c}
n \\
i
\end{array}\right) p^{i}(1-p)^{n-i}
$$

Using the idea of conditional probability of accepting lot with $C_{1}$, given sample $n$, the resulting probability of lot acceptance or Operating Characteristic (OC) function is given as:

$$
P a=\frac{\sum_{\mathrm{i}=0}^{\mathrm{c}_{1}\left(\begin{array}{l}
\mathrm{n} \\
\mathrm{i}
\end{array}\right)(1-p)^{\mathrm{n}-\mathrm{i}}}}{\sum_{\mathrm{i}=0}^{\mathrm{c}_{1}}\left(\begin{array}{l}
\mathrm{n} \\
\mathrm{i}
\end{array}\right)(1-p)^{\mathrm{n}-\mathrm{i}}+\left(1-\sum_{\mathrm{i}=0}^{\mathrm{c}_{2}}\left(\begin{array}{l}
\mathrm{n} \\
\mathrm{i}
\end{array}\right) \mathrm{p}^{\mathrm{i}}(1-\mathrm{p})^{\mathrm{n}-\mathrm{i}}\right)}
$$

where $p$ is the probability that an item is defective or lot fraction defective under a given product life distribution. Therefore, the operating characteristic formula for our proposed plan becomes:

$$
P a=\frac{\mathrm{Pa}}{\mathrm{Pa}+(1-\mathrm{Pr})}
$$

The failure probabilities are represented by the cumulative distribution function of the life time distributions.

\subsection{Mean Life Ratio Value}

In order to calculate the minimum required ratio values, the producer's risk is been considered. The producer's risk is the probability of rejection of the lot when $\mu \geq \mu_{0}$, it can be computed as follows;

$$
\begin{gathered}
\operatorname{Pr}(R)=\mathrm{P}(\text { Rejecting a lot })=1-P\left(\text { AcceptingtheLot } / \mu \geq \mu_{0}\right) \\
=\sum_{i=c+1}^{n}\left(\begin{array}{c}
n \\
i
\end{array}\right) p^{i}(1-p)^{n-1}
\end{gathered}
$$

For the given sampling plan and for a given value of the producer's risk, say $\gamma$, one may beinterested in knowing the minimum value of $\frac{\mu}{\mu_{0}}$, that will ensure the producer's risk to beat most $\gamma$. The $\frac{\mu}{\mu_{0}}$, is the smallest quantity for which $P$ satisfies the inequality.

\section{Results and Analysis}

Suppose the lifetime of the testing items follow a Weibull distribution with known shape parameter, the numerical values that will serve as guide to the tester is presented in the in tables 2 to 4 .

Figures 2 is the OC plots of probability of acceptance $(\mathrm{Pa})$ against mean ratio $\left(\frac{\mu}{\mu_{0}}\right)$ for fixed $\frac{t}{\mu_{0}}=0.942,1.257,1.571,2.358,3.141$ and $3.972 \quad$ and varying $\beta=0.25,0.10,0.05,0.01$. For fixed $\beta=0.05$ and varying $\frac{t}{\mu_{0}}$, figure 3 depicts the OC curves.

In order to compare our results with existing researches, we used existing combined parameters to simulate our results using $R$ Software.

\subsection{Sensitivity Analysis}

In statistical quality control, operating characteristic curves plays an important role in determining the probability of accepting manufactured lots when using different sampling plans. It shows the relationship between a designed parameter and lot acceptance when we conduct a lifetime experiment. The OC curves helps in the selection of acceptance sampling plans and also help in reducing risks. The different behavior of OC values and combined parameters are presented in Figures 2 to 5. Thus, after analyzing the trends of the results given in Tables 3 to 5, one can make the trade-off between the required minimum sample size, confidence level, acceptance number and experimental time ratio to achieve the best sampling plan.

\subsection{Real Life Example}

The data used in this study were collected from the quality assurance and assembly plant of machine and tool department, Udofe metal industries, KM 3, Igarra, Okpe Road, Edo State, Nigeria. The data are the approximate number of revolutions (millions) of Oil Palm Milling Machine Ball-Bearings before failure. The data as retrieved from the record file of the Quality Assurance Department of the Industry are: $28.44,28.16,29.22,32.56,30.83,27.44$, 26.64, 34.88, 29.02, 30.42, 29.61, 30.02, 28.94, 31.94, 30.04, 29.79, 27.20, 33.54, 31.45 and 29.23.

Suppose a manufacturer want to develop a Sampling Plan and know whether the life of his products(ball bearing) are above the specified mean life revolution of 30 million revolutions per hour with Maximum Allowable Percent Defective $(\beta=0.10)$ and the life test would be ended at 25 million revolutions, which should have led to the ratio $\frac{t}{\mu_{o}}=0.833$. Consider that the lifetime of products follows a 
Weibull distribution. Thus, from Table 2, Braimah and Osanaiye (2016), for an acceptance number $\mathrm{C}=2$, the designed parameters of the Sampling Plan are $\left(n, C, \frac{t}{\mu_{o}}\right)=$ 3,2 and 0.833 for $\beta=0.10$. That is the manufacturer needs to select a sample of 3 products and put on test, the lot is rejected if more than 2 failures occur during 25 million revolution test per hour, otherwise accept it.

The OC values for the acceptance sampling plan $\left(n, C, \frac{t}{\mu_{o}}\right)=3,2$ and 0.833 for $\beta=0.10$ as extracted from Table 4 for a Weibull product life distribution with $\frac{\mu}{\mu_{0}}=2$ is as shown in Table 2 below.

Table 1. OC values for the acceptance sampling plan $\left(n, C, \frac{t}{\mu_{o}}\right)=$ 3,2 and 0.833 for $\beta=0.10$ under Weibull distribution with $\frac{\mu}{\mu_{0}}=2$.

\begin{tabular}{lllllll}
\hline$\frac{\boldsymbol{\mu}}{\boldsymbol{\mu}_{\mathbf{0}}}$ & $\mathbf{2}$ & $\mathbf{4}$ & $\mathbf{6}$ & $\mathbf{8}$ & $\mathbf{1 0}$ & $\mathbf{1 2}$ \\
\hline 0.628 & 0.9999 & 1.0000 & 1.0000 & 1.0000 & 1.0000 & 1.0000 \\
\hline
\end{tabular}

It can deduce from the above table that if the true mean life is twice the required mean life $\left(\frac{\mu}{\mu_{0}}=2\right)$, the producer'srisk is approximately $1-0.68035=0.0001$.

From Table 5, the experimenter can get the values of mean life ratio for different choices of $c$ and $\frac{t}{\mu_{o}}$ in order to assert that the producer's risk was less than 0.05 . In this example, the mean life ratiovalue of his product should be 3.049 for $c$ $=2, \frac{\boldsymbol{t}}{\mu_{0}}=0.942$ and $\beta=0.10$. This means the product can have a mean life of 3.049 times the required mean lifetime in order that under the above acceptance sampling plan the product is accepted with probability of at least 0.90 .

\section{Discussion of Results}

This section interprets our observation from the simulated results and operating characteristics plots using $\mathrm{R}$ software as shown in Table 2 to 4 and Figure 2 to 4 below.

\subsection{Interpretation of the Behaviour of Operating Characteristics}

From Table 4, the following can be deduced:

i. On fixing the experiment time ratio and varying mean ratio, the probability of acceptance is decreasing with an increase in the confidence level. We also observed the same trend in respect of experiment time ratio for a fixed confidence level.

ii. On fixing the confidence level and experiment time ratio, the probability of acceptance increases as the mean ratio increases.

\subsection{Interpretation for the Minimum Required Mean Ratio at Fixed Producer's Risk}

From Table 4, the following can be deduced:

i. It was observed that the minimum mean ratios required for smaller acceptance number in order that the lot will be accepted with the probability $(1-\alpha)$ are very high as compared to higher acceptance number for any combination of confidence level and experiment time ratio.

ii. On fixing the acceptance number, the required minimum means ratio increases as the confidence level increases.

\subsection{Interpretation of Operating Characteristics Curves}

i. From Figure 3, for any fixed value of Maximum Allowable Percent Defectives $(\beta)$ and experiment time ratio, the $\mathrm{OC}$ values of Weibill product life distribution increases as the mean life ratio increases.

ii. From figure 4, for any fixed value of maximum allowable percent defectives $(\beta)$ and experiment time ratio, the $\mathrm{OC}$ values of Weibill product life distribution also increases as the experimental ratio increases. This may happen due to the incorporation of the past parametric fluctuations with the experimental data.

iii. From figure 4, our proposed plan resulted to smaller consumers' risk than the when compares with the work of Aslam and Ahmad (2009).

\subsection{Comparison of Our Proposed Plan with Existing Truncated Double Sampling Plan}

In order compare the performance of our proposed sampling plan (Truncated Hybrid Double Acceptance Sampling Plan) with the ordinary Truncated Double Sampling Plan by Aslam and Ahmad (2009), we plotted the product mean ratio against the probability of acceptance (OC) values. This is as shown in the OC plot in Figure 4 below.

From the OC plot in Figure 4, the proposed hybrid truncated double sampling plan has the optimal probability of acceptance than the ordinary double sampling plan.

\section{Conclusion}

In this study, a Truncated Hybrid Double Acceptance Sampling Plan for the Weibull product life distribution was proposed. It is assumed that the shape parameter is known and we have presented the results in tables for the developed minimum sample size required to guarantee a certain mean life of the test units.

Conclusively, our results can also serve for other product life distributions that belong to the family of Weibull distribution. Therefore, our tables can be used to develop the acceptance sampling plan for these product life distributions that will reduce testing time, cost and minimize the producer and consumers' risk and on the other hand, guide the producer in improving his product life.

The design parameters for this proposed plan were determined by the two point approach considering both the producer's $(\alpha)$ and the consumer's risks $(\beta)$ simultaneously. The quality level of an item (product) was then considered in terms of the mean life ratio to the specified life.

Our simulated results are presented in Table 2 to 4 with real life example to illustrate the results. 
Table 2. Developed minimum sample size for Weibull distribution and the corresponding acceptance number $c$ when the shape parameter $\alpha=2$.

\begin{tabular}{|c|c|c|c|c|c|c|c|c|c|c|}
\hline & & & $\frac{t}{\mu_{0}}$ & & & & & & & \\
\hline$\beta$ & & $c$ & 0.628 & 0.942 & 1.257 & 1.571 & 2.356 & 3.141 & 3.972 & 4.713 \\
\hline \multirow{10}{*}{0.25} & 0 & & 2 & 2 & 2 & 2 & 2 & 2 & 1 & 1 \\
\hline & 1 & & 3 & 3 & 3 & 2 & 2 & 2 & 2 & 2 \\
\hline & 2 & & 4 & 4 & 3 & 3 & 3 & 3 & 3 & 2 \\
\hline & 3 & & 5 & 5 & 4 & 4 & 3 & 3 & 3 & 3 \\
\hline & 4 & & 6 & 6 & 4 & 4 & 4 & 4 & 4 & 4 \\
\hline & 5 & & 7 & 7 & 5 & 5 & 4 & 4 & 4 & 4 \\
\hline & 6 & & 8 & 8 & 5 & 5 & 5 & 5 & 5 & 5 \\
\hline & 7 & & 8 & 8 & 6 & 6 & 5 & 5 & 5 & 5 \\
\hline & 8 & & 9 & 9 & 7 & 7 & 6 & 6 & 6 & 6 \\
\hline & 9 & & 10 & 10 & 7 & 7 & 6 & 6 & 6 & 6 \\
\hline \multirow{12}{*}{0.10} & 10 & & 11 & 11 & 8 & 8 & 7 & 7 & 7 & 7 \\
\hline & 0 & & 2 & 2 & 2 & 2 & 2 & 2 & 2 & 1 \\
\hline & 1 & & 3 & 3 & 3 & 3 & 2 & 2 & 3 & 3 \\
\hline & 2 & & 4 & 4 & 3 & 3 & 3 & 3 & 3 & 3 \\
\hline & 3 & & 5 & 5 & 5 & 4 & 4 & 4 & 4 & 4 \\
\hline & 4 & & 6 & 6 & 5 & 4 & 4 & 4 & 4 & 4 \\
\hline & 5 & & 7 & 7 & 5 & 5 & 4 & 4 & 4 & 4 \\
\hline & 6 & & 8 & 8 & 5 & 5 & 5 & 5 & 5 & 5 \\
\hline & 7 & & 8 & 8 & 6 & 6 & 5 & 5 & 5 & 5 \\
\hline & 8 & & 9 & 9 & 8 & 7 & 6 & 6 & 6 & 6 \\
\hline & 9 & & 11 & 10 & 9 & 7 & 6 & 6 & 6 & 6 \\
\hline & 10 & & 11 & 11 & 9 & 8 & 7 & 6 & 6 & 6 \\
\hline \multirow{10}{*}{0.05} & 0 & & 3 & 3 & 3 & 3 & 3 & 3 & 2 & 2 \\
\hline & 1 & & 5 & 5 & 5 & 5 & 4 & 4 & 4 & 3 \\
\hline & 2 & & 5 & 5 & 5 & 5 & 5 & 5 & 4 & 4 \\
\hline & 3 & & 7 & 7 & 6 & 6 & 6 & 6 & 6 & 4 \\
\hline & 4 & & 7 & 7 & 7 & 6 & 6 & 6 & 6 & 4 \\
\hline & 5 & & 7 & 7 & 7 & 7 & 6 & 6 & 6 & 5 \\
\hline & 6 & & 7 & 7 & 7 & 7 & 6 & 6 & 6 & 6 \\
\hline & 7 & & 8 & 8 & 8 & 7 & 6 & 6 & 6 & 6 \\
\hline & 8 & & 8 & 8 & 8 & 7 & 7 & 7 & 7 & 7 \\
\hline & 9 & & 9 & 8 & 8 & 7 & 7 & 7 & 7 & 7 \\
\hline \multirow{12}{*}{0.01} & 10 & & 9 & 8 & 8 & 8 & 8 & 7 & 7 & 8 \\
\hline & 0 & & $\mathrm{c}$ & 4 & 4 & 3 & 3 & 3 & 3 & 2 \\
\hline & 1 & & 4 & 4 & 4 & 4 & 4 & 3 & 3 & 3 \\
\hline & 2 & & 5 & 5 & 5 & 5 & 4 & 4 & 3 & 3 \\
\hline & 3 & & 5 & 5 & 4 & 4 & 4 & 4 & 4 & 4 \\
\hline & 4 & & 6 & 6 & 4 & 4 & 4 & 4 & 4 & 4 \\
\hline & 5 & & 7 & 7 & 5 & 5 & 5 & 5 & 5 & 5 \\
\hline & 6 & & 8 & 8 & 5 & 5 & 5 & 5 & 5 & 5 \\
\hline & 7 & & 8 & 8 & 6 & 6 & 5 & 5 & 5 & 5 \\
\hline & 8 & & 9 & 9 & 7 & 7 & 6 & 6 & 6 & 6 \\
\hline & 9 & & 10 & 10 & 7 & 7 & 6 & 6 & 6 & 6 \\
\hline & 10 & & 11 & 11 & 8 & 8 & 6 & 6 & 7 & 7 \\
\hline
\end{tabular}


Table 3. Design parameters of the proposed Truncated Hybrid Double Acceptance Sampling Plan for the Weibull distribution with $\alpha=2$.

\begin{tabular}{|c|c|c|c|c|c|c|c|c|c|c|c|}
\hline \multirow[t]{2}{*}{$\boldsymbol{\beta}$} & \multirow{2}{*}{$\frac{\mu}{\mu_{o}}$} & \multirow[t]{2}{*}{$\mathrm{C}_{1}$} & \multirow[t]{2}{*}{$\mathrm{C}_{2}$} & \multirow[t]{2}{*}{$n$} & \multicolumn{7}{|l|}{$\frac{t}{\mu_{o}}$} \\
\hline & & & & & 0.628 & 0.942 & 1.571 & 2.356 & 3.141 & 3.972 & 4.713 \\
\hline \multirow{6}{*}{0.25} & 2 & 0 & 1 & 3 & 0.9678 & 0.8331 & 0.2627 & 0.0181 & 0.0006 & 0.0000 & 0.0000 \\
\hline & 4 & 1 & 2 & 4 & 0.9999 & 0.9994 & 0.9885 & 0.8941 & 0.5945 & 0.2226 & 0.0640 \\
\hline & 6 & 2 & 3 & 5 & 1.0000 & 1.0000 & 0.9999 & 0.9981 & 0.9855 & 0.9303 & 0.8017 \\
\hline & 8 & 3 & 4 & 6 & 1.0000 & 1.0000 & 1.0000 & 1.0000 & 0.9997 & 0.9975 & 0.9896 \\
\hline & 10 & 4 & 5 & 7 & 1.0000 & 1.0000 & 1.0000 & 1.0000 & 1.0000 & 0.9999 & 0.9996 \\
\hline & 12 & 5 & 6 & 8 & 1.0000 & 1.0000 & 1.0000 & 1.0000 & 1.0000 & 1.0000 & 1.0000 \\
\hline \multirow{6}{*}{0.10} & 2 & 0 & 1 & 3 & 0.9678 & 0.8331 & 0.2627 & 0.0181 & 0.0006 & 0.0000 & 0.0000 \\
\hline & 4 & 1 & 2 & 4 & 0.9999 & 0.9994 & 0.9885 & 0.8941 & 0.5945 & 0.2226 & 0.0640 \\
\hline & 6 & 2 & 3 & 5 & 1.0000 & 1.0000 & 0.9999 & 0.9981 & 0.9855 & 0.9303 & 0.8017 \\
\hline & 8 & 3 & 4 & 6 & 1.0000 & 1.0000 & 1.0000 & 1.0000 & 0.9997 & 0.9975 & 0.9896 \\
\hline & 10 & 4 & 5 & 7 & 1.0000 & 1.0000 & 1.0000 & 1.0000 & 1.0000 & 0.9999 & 0.9996 \\
\hline & 12 & 5 & 6 & 8 & 1.0000 & 1.0000 & 1.0000 & 1.0000 & 1.0000 & 1.0000 & 1.0000 \\
\hline \multirow{6}{*}{0.05} & 2 & 0 & 1 & 3 & 0.9355 & 0.6968 & 0.1192 & 0.0041 & 0.0001 & 0.0000 & 0.0000 \\
\hline & 4 & 1 & 2 & 4 & 0.9999 & 0.9985 & 0.9733 & 0.7787 & 0.3616 & 0.0854 & 0.0170 \\
\hline & 6 & 2 & 3 & 5 & 1.0000 & 1.0000 & 0.9997 & 0.9949 & 0.9631 & 0.8385 & 0.6065 \\
\hline & 8 & 3 & 4 & 6 & 1.0000 & 1.0000 & 0.9999 & 0.9986 & 0.9898 & 0.9549 & 0.8826 \\
\hline & 10 & 4 & 5 & 7 & 1.0000 & 1.0000 & 1.0000 & 1.0000 & 1.0000 & 0.9999 & 0.9996 \\
\hline & 12 & 5 & 6 & 8 & 1.0000 & 1.0000 & 1.0000 & 1.0000 & 1.0000 & 1.0000 & 1.0000 \\
\hline \multirow{6}{*}{0.01} & 2 & 0 & 1 & 3 & 0.9355 & 0.6968 & 0.1192 & 0.0041 & 0.0001 & 0.0000 & 0.0000 \\
\hline & 4 & 1 & 2 & 4 & 0.9999 & 0.9985 & 0.9733 & 0.7787 & 0.3616 & 0.0854 & 0.0170 \\
\hline & 6 & 2 & 3 & 5 & 1.0000 & 1.0000 & 0.9999 & 0.9981 & 0.9855 & 0.9303 & 0.8017 \\
\hline & 8 & 3 & 4 & 6 & 1.0000 & 1.0000 & 1.0000 & 1.0000 & 0.9997 & 0.9975 & 0.9896 \\
\hline & 10 & 4 & 5 & 7 & 1.0000 & 1.0000 & 1.0000 & 1.0000 & 1.0000 & 0.9999 & 0.9996 \\
\hline & 12 & 5 & 6 & 8 & 1.0000 & 1.0000 & 1.0000 & 1.0000 & 1.0000 & 1.0000 & 1.0000 \\
\hline
\end{tabular}

Table 4. Minimum ratio of true mean life to specified mean for acceptance of lot of when the life time of a product follows a Weibull distribution.

\begin{tabular}{|c|c|c|c|c|c|c|c|c|c|}
\hline & & $\frac{t}{\mu_{0}}$ & & & & & & & \\
\hline$\beta$ & $c$ & 0.628 & 0.942 & 1.257 & 1.571 & 2.356 & 3.141 & 3.972 & 4.713 \\
\hline \multirow{14}{*}{0.25} & 0 & 6.188 & 7.177 & 7.797 & 9.746 & 10.271 & 13.694 & 17.118 & 20.541 \\
\hline & 2 & 2.363 & 2.666 & 2.955 & 3.252 & 4.097 & 5.462 & 5.142 & 6.171 \\
\hline & 3 & 2.067 & 2.244 & 2.571 & 2.575 & 3.264 & 4.352 & 4.147 & 4.976 \\
\hline & 4 & 1.898 & 2.116 & 2.169 & 2.463 & 2.780 & 3.706 & 4.632 & 4.269 \\
\hline & 5 & 1.787 & 1.941 & 2.060 & 2.165 & 2.884 & 3.275 & 4.094 & 3.793 \\
\hline & 6 & 1.709 & 1.818 & 1.981 & 2.142 & 2.603 & 2.963 & 3.704 & 4.444 \\
\hline & 7 & 2.394 & 2.381 & 2.362 & 2.338 & 2.350 & 2.291 & 2.314 & 2.644 \\
\hline & 8 & 2.257 & 2.251 & 2.218 & 2.229 & 2.197 & 2.186 & 2.171 & 2.478 \\
\hline & 9 & 2.150 & 2.150 & 2.140 & 2.110 & 2.076 & 2.100 & 2.058 & 2.356 \\
\hline & 10 & 2.081 & 2.067 & 2.048 & 2.044 & 2.026 & 1.986 & 1.969 & 2.251 \\
\hline & 0 & 7.327 & 8.297 & 9.570 & 9.746 & 14.619 & 13.694 & 17.118 & 20.541 \\
\hline & 1 & 3.699 & 3.970 & 4.368 & 4.777 & 5.956 & 7.941 & 9.926 & 8.781 \\
\hline & 2 & 2.829 & 3.049 & 3.269 & 3.694 & 4.877 & 5.462 & 6.828 & 8.193 \\
\hline & 3 & 2.391 & 2.645 & 2.790 & 3.214 & 3.862 & 4.352 & 5.440 & 6.528 \\
\hline \multirow{11}{*}{0.10} & 5 & 2.026 & 2.190 & 2.340 & 2.575 & 3.247 & 3.846 & 4.094 & 4.912 \\
\hline & 6 & 1.909 & 2.031 & 2.214 & 2.476 & 2.926 & 3.471 & 3.704 & 4.444 \\
\hline & 7 & 1.750 & 1.850 & 1.950 & 1.970 & 2.400 & 2.740 & 2.730 & 3.280 \\
\hline & 8 & 1.670 & 1.760 & 1.800 & 1.970 & 2.230 & 2.560 & 2.580 & 3.100 \\
\hline & 9 & 1.630 & 1.690 & 1.770 & 1.840 & 2.090 & 2.420 & 2.450 & 2.940 \\
\hline & 10 & 1.600 & 1.680 & 1.740 & 1.731 & 1.980 & 2.300 & 2.340 & 2.810 \\
\hline & 0 & 8.762 & 9.282 & 11.062 & 11.962 & 14.619 & 19.492 & 24.365 & 20.541 \\
\hline & 1 & 3.988 & 4.557 & 4.853 & 5.460 & 7.165 & 7.941 & 9.926 & 11.911 \\
\hline & 2 & 2.995 & 3.222 & 3.555 & 4.087 & 4.877 & 5.462 & 6.828 & 8.193 \\
\hline & 3 & 2.567 & 2.766 & 2.992 & 3.214 & 3.862 & 5.149 & 5.440 & 6.528 \\
\hline & 4 & 6.188 & 7.177 & 7.797 & 9.746 & 10.271 & 13.694 & 17.118 & 20.541 \\
\hline \multirow{6}{*}{0.05} & 5 & 3.038 & 3.276 & 3.821 & 3.971 & 5.956 & 5.854 & 7.318 & 8.781 \\
\hline & 6 & 2.363 & 2.666 & 2.955 & 3.252 & 4.097 & 5.462 & 5.142 & 6.171 \\
\hline & 7 & 2.067 & 2.244 & 2.571 & 2.575 & 3.264 & 4.352 & 4.147 & 4.976 \\
\hline & 8 & 1.898 & 2.116 & 2.169 & 2.463 & 2.780 & 3.706 & 4.632 & 4.269 \\
\hline & 9 & 1.787 & 1.941 & 2.060 & 2.165 & 2.884 & 3.275 & 4.094 & 3.793 \\
\hline & 10 & 1.709 & 1.818 & 1.981 & 2.142 & 2.603 & 2.963 & 3.704 & 4.444 \\
\hline
\end{tabular}




\begin{tabular}{|c|c|c|c|c|c|c|c|c|c|}
\hline & & $\frac{t}{\mu_{0}}$ & & & & & & & \\
\hline \multirow[t]{10}{*}{$\boldsymbol{\beta}$} & $c$ & 0.628 & 0.942 & 1.257 & 1.571 & 2.356 & 3.141 & 3.972 & 4.713 \\
\hline & 2 & 2.150 & 2.150 & 2.140 & 2.110 & 2.076 & 2.100 & 2.058 & 2.356 \\
\hline & 3 & 2.081 & 2.067 & 2.048 & 2.044 & 2.026 & 1.986 & 1.969 & 2.251 \\
\hline & 4 & 7.327 & 8.297 & 9.570 & 9.746 & 14.619 & 13.694 & 17.118 & 20.541 \\
\hline & 5 & 3.699 & 3.970 & 4.368 & 4.777 & 5.956 & 7.941 & 9.926 & 8.781 \\
\hline & 6 & 2.829 & 3.049 & 3.269 & 3.694 & 4.877 & 5.462 & 6.828 & 8.193 \\
\hline & 7 & 2.391 & 2.645 & 2.790 & 3.214 & 3.862 & 4.352 & 5.440 & 6.528 \\
\hline & 8 & 2.192 & 2.320 & 2.517 & 2.711 & 3.274 & 3.706 & 4.632 & 5.559 \\
\hline & 9 & 2.026 & 2.190 & 2.340 & 2.575 & 3.247 & 3.846 & 4.094 & 4.912 \\
\hline & 10 & 1.909 & 2.031 & 2.214 & 2.476 & 2.926 & 3.471 & 3.704 & 4.444 \\
\hline
\end{tabular}

Operating Characteristics Curves

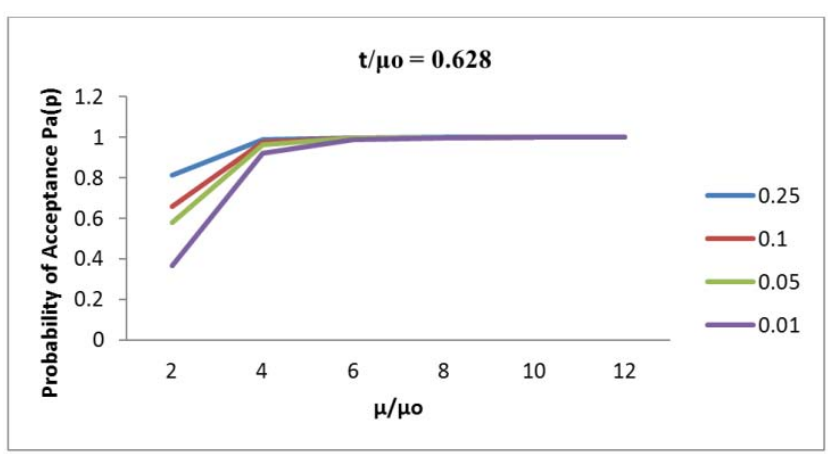

Figure 2. Operating characteristics curve of probability of acceptance against mean life ratios for various Maximum Allowable Percent Defective (MAPD).

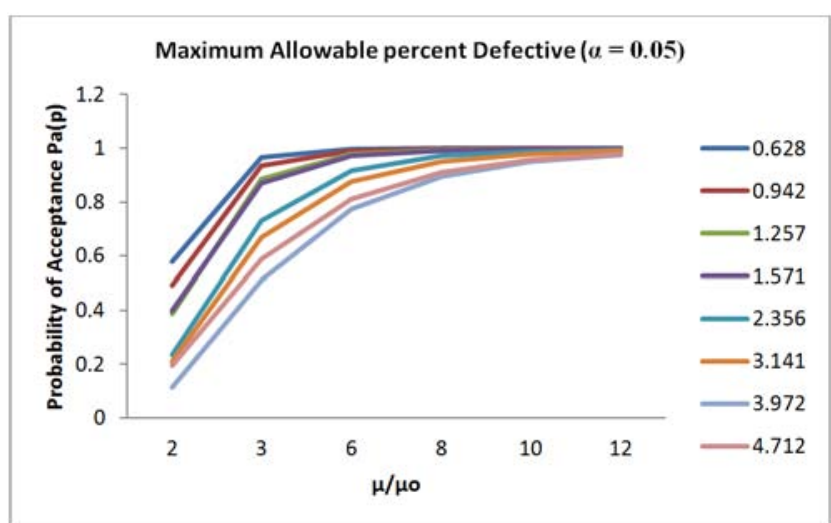

Figure 3. Operating characteristics curve of probability of acceptance against experimental mean ratios at various experimental time ratios.

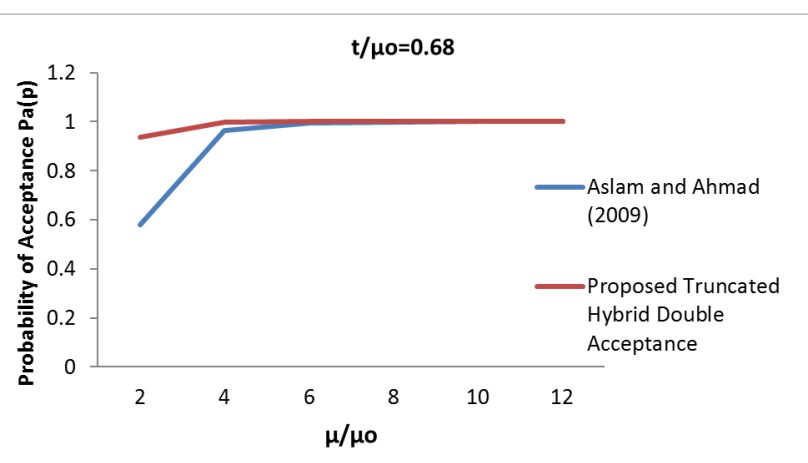

Figure 4. Operating Characteristics plot with respect to experiment time ratio when Maximum Allowable percent Defective $\alpha=0.25$ and $\frac{t}{\mu_{o}}=$ 0.628 .

\section{References}

[1] Aslam, M. and R. R. L. Kantam (2008). Economic acceptance sampling based on truncated life tests in the BirnbaumSaunders distribution. Pak. J. Stat., 24(4): pp.269-276.

[2] Aslam, M. and C. H. Jun (2009). A group acceptance sampling plan for truncated life test having Weibull distribution. J. Appl. Stat., 36(9): pp.1021-1027.

[3] Aslam, M., C. H. Jun and M. Ahmad (2009). Double acceptance sampling plans based on truncated life tests in the Weibull model. J. Stat. Theor. Appl., 8(2): pp. 191-206.

[4] Balakrishnan.M, Leiva.V and Lopez.J (2007). Acceptance sampling plans from truncatedlife tests based on the Generalized Birnbaum-Saunders distribution. Comm. Stat. Simul. Comp., (36), pp. 643-656

[5] Balamurali, S. and C.H. Jun (2006). Repetitive group sampling procedure for variables inspection. J. Appl. Stat., 33(3): pp. 327-338.

[6] Braimah, O. J and Osanaiye, P. A. (2016). Improved single truncated acceptance sampling plans for product dife Distributions, Journal of Sustainable Development in Africa, 18(3):pp. 91-115.

[7] Braimah O. J, Osanaiye P. A and Edokpa I. W. (2016). Improved single truncated acceptance sampling plans for Weibull product life distributions.Journal of the National Association of Mathematical Physics, 38, pp. 451-460.

[8] Epstein, B. (1954). Truncated life tests in the Exponential Case. Ann. Math. Statist. (25), pp.555-564 Goode.

[9] H. P. and Kao, J. H. K. (1961). Sampling plans based on the Weibull distribution. In Proc 7th Nat. Symp. Rel. Qual. Cont., pp. $24-40$.

[10] Gupta S. S. (1960). Order Statistics from Gamma Distribution. Technometrics, (2), pp. 243-262.

[11] Gupta S. S. (1962). Life Test sampling plans for normal and lognormal distributions. Technometrics, 4(2), pp. 151-175.

[12] Marshall, A. W and Olkin, I. (1997). A new method for adding a parameter to a family of distributions with application to the exponential and Weibull families. Biometrika, 84, pp. 641652.

[13] Muhammad, A., Debasis, K. and Munir, A. (2010). Time truncated acceptance sampling plans for Generalized Exponential distribution.Pak. J. Commer. Soc. Sci. 1, pp.1-20. 
[14] Priyah and Ramaswamy, A. R. S. (2015). A group acceptance aamplingPpan for weighted binomial on truncated life tests using Exponential and Weibull distributions. Journal of Progressive Research in Mathematics. 2(1), pp. 80-88.

[15] Sherman, R. E. (1965). Design and evaluation of repetitive group sampling plan. Technometrics, pp. 11-21.

[16] Sobel, M. and Tischendr of, J. A. (1959).Acceptance sampling with new life test objectives. Proceedings of Fifth National Symposium on Reliability and Quality Cont., 1, pp. 108-118.

[17] Srinivasa S. (2011). Double acceptance sampling plans based on truncated life tests for the Marshall Olkin's extended exponential distribution, Austrian Journal of Statistics, 40(3), pp. 169-176.
[18] Sudamani, A. R. R and Priyah A. (2012).Acceptance sampling plan for truncated life tests at maximum allowable percent defective. Int. J. of Computational Engr. Research.,2(5), pp. 1413-1418.

[19] Sudamani, A. R. R. and Jayasri, S. (2012). Time truncated chain pampling plans for generalized exponential distribution. Int. J. of Computational Engr. Research (ijceronline.com). 2 (5), pp.1402-1407.

[20] Sudamani, A. R. R. and Jayasri S. (2013).Time truncated chain sampling plans for Marshall-Olkin extended exponential distributions. IOSR J. Of Maths., 5(1), pp. 01-05.

[21] Sudamani, R. R and Jayasri, S (2016). Time truncated chain sampling plan for Welbull distributions. International Journal of Engineering Research and General Science, 3(2).pp.59-67. 